\title{
An Investigation of Structural Damage Location Based on Ultrasonic Excitation-Fiber Bragg Grating Detection
}

\author{
Yuegang Tan, Li Cai, Bei Peng, and Lijun Meng \\ School of Mechanical and Electronic Engineering, Wuhan University of Technology, Wuhan 430070, China \\ Correspondence should be addressed to Li Cai; caili_whut@163.com
}

Received 15 March 2013; Accepted 18 August 2013

Academic Editor: K. M. Liew

Copyright ( 2013 Yuegang Tan et al. This is an open access article distributed under the Creative Commons Attribution License, which permits unrestricted use, distribution, and reproduction in any medium, provided the original work is properly cited.

\begin{abstract}
With the continuous development of mechanical automation, the structural health monitoring techniques are increasingly high requirements for damage detection. So structural health monitoring (SHM) has been playing a significant role in terms of damage prognostics. The main contribution pursued in this investigation is to establish a detection system based on ultrasonic excitation and fiber Bragg grating sensing, which combines the advantages of the ultrasonic detection and fiber Bragg grating (FBG). Differencing from most common approaches, a new way of damage detection is based on fiber Bragg grating (FBG), which can easily realize distributed detection. The basic characteristics of fiber Bragg grating sensing system are analyzed, and the positioning algorithm of structural damage is derived in theory. On these bases, the detection system was used to analyze damage localization in the aluminum alloy plate of a hole with diameters of $6 \mathrm{~mm}$. Experiments have been carried out to demonstrate that the sensing system was feasible and that the estimation method of the location algorithm was easy to implement.
\end{abstract}

\section{Introduction}

Recently, structural health monitoring (SHM) has been playing a significant complimentary role in terms of damage prognostics, which has been applied in many fields, especially in aerospace, civil engineering, railway field extensions, and even in the automobile industry. Traditional nondestructive evaluation (NDE) techniques include ultrasonic, eddy current, and magnetic particle. In recent years, SHM using ultrasonic excitation and piezoelectric ceramics network has been extensively studied, while ultrasonic excitation and fiber Bragg grating sensing technology is a new damage detection method [1]. It fully combines ultrasonic advantages, such as focused power, strong sound press, and long propagation length and the characteristic that FBG can easily construct distributed detection network, overcoming the disadvantages of traditional electronic sensors.

At present, it has already been detected by related researchers. Such as, Tsuda presented a novel ultrasound sensing system using a fiber Bragg grating (FBG) and broadband light source to damage inspection [2] and measured imparting damage to carbon fiber composite using FBG [3]. Brian Culshaw summarized the interaction mechanisms between ultrasound and fiber sensors and confirms their functional flexibility to detect damage in a sample [4]. Tsuda et al. analyzed ultrasonic sensitivity through an aluminum plate and also evaluated from the response the amplitude of the FBG sensor [5]. Jang et al. used multiplexed fiber Bragg grating (FBG) sensor to study positioning algorithm for composite structures $[6,7]$. However, these predicted detections often differ from real damage. Only a few nondestructive testing techniques can be considered sufficiently for smart materials. The method involves the analysis of the transmitted and reflected waves. The presence of damage is identified by contrasting the detected signal with the reference signal.

Aiming at the application of FBG sensors in ultrasonic testing, this paper focuses on the use of FBGs as ultrasonic receivers. The intention of the paper is to present a FBG sensing system based on ultrasonic excitation for the board plates. The structure of the paper is as follows: in Section 2, the FBG sensing system is introduced briefly, and then the characteristics of the FBG sensing signals under ultrasonic excitation are analyzed, and Section 3 analyzes the damage localization algorithm based on elliptical technology, whose practical implementation is described using an aluminum alloy plate with a hole with diameters of $6 \mathrm{~mm}$ in Section 4. Finally, the testing results obtained using this estimation method are given and the paper is concluded in Section 5. 


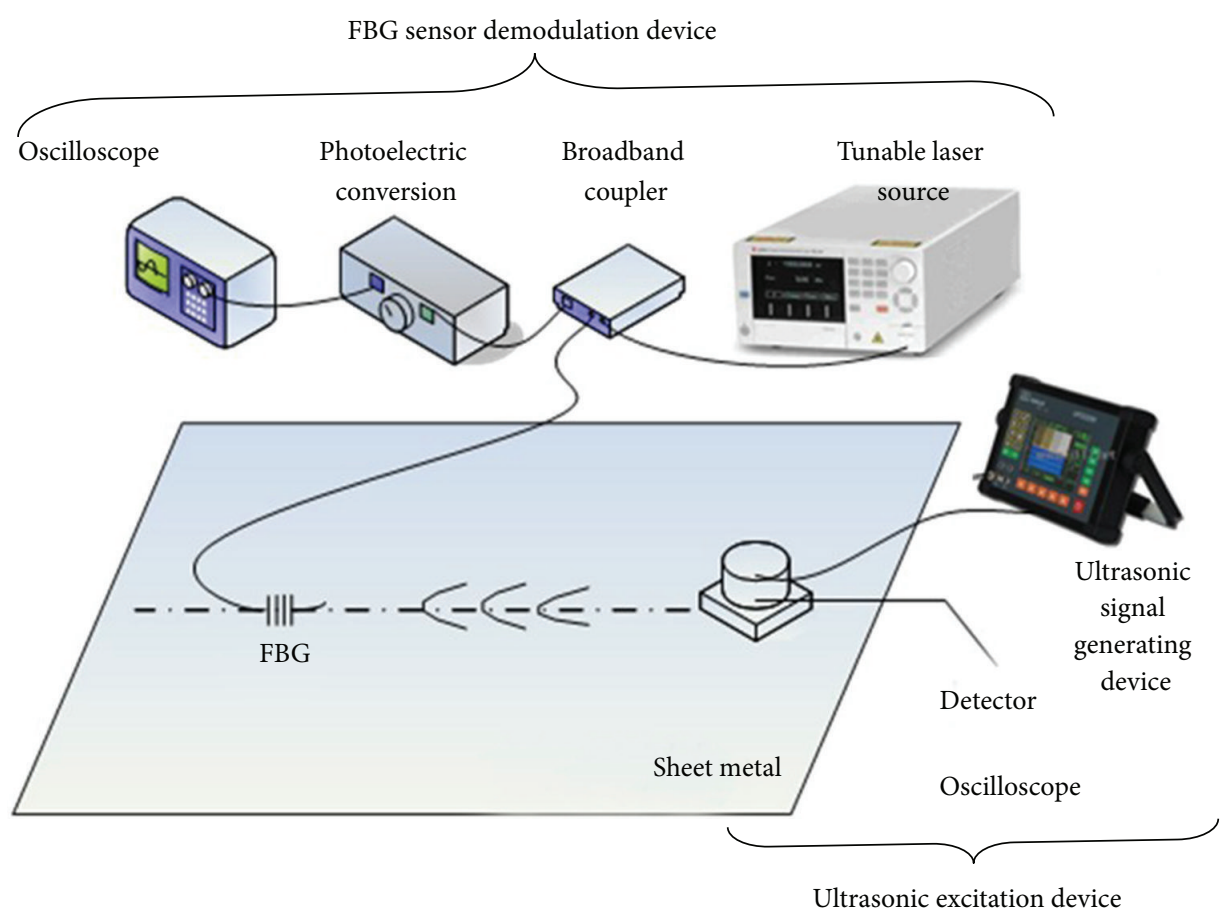

FIGURE 1: Detection system based on ultrasonic excitation and FBG sensing.

\section{Basic Characteristics of the Ultrasonic Excitation and FBG Sensing}

The strain caused by ultrasonic spreading in the material is small, usually only a few or a dozen microstrains, so it should be ensured that fiber grating demodulation system has a high demodulation speed to realize the measurement of ultrasonic signals. In addition, the optical fiber grating has an axial strain sensitivity, while the ultrasonic energy is focused on the acoustic axis. In the far field region, the sound on the axis of the sound pressure is reduced with the increase of the relative sound-source distance. To this end, we first study the basic characteristics of ultrasonic excitation and fiber Bragg grating sensing.

The objects of study are three different materials (steel, copper, and aluminum) plates. Experimental measurement system is shown in Figure 1, and the signal demodulated method is an adjustable laser edge filter demodulation. The plate's dimensions are all $300 \mathrm{~mm} * 300 \mathrm{~mm} * 2 \mathrm{~mm}$, FBG paste location diagram is shown in Figure 2, FBG transversely paste and the wavelength is $1305.304 \mathrm{~nm}$.

\subsection{Research on Ways of Ultrasonic Excitation and Medium.} Experimental object is aluminum plate, which has 15 test points in each plate and is shown in Figure 2. The experimental data should be saved in disk, and analyzed by matlab software. Then, each test point of the received signal $V_{\mathrm{pp}}$ (signal peak-peak) is obtained. The following diagram in Figure 3 is the changing curve of ultrasonic intensity on aluminum plate by lame wave. it can be obviously seen that $V_{\text {pp }}$ decreased significantly with the angle and the distance increasing. It can be seen from Figure 4 that the use of the detection results by lame wave is more preferable in ultrasonic damage detection.

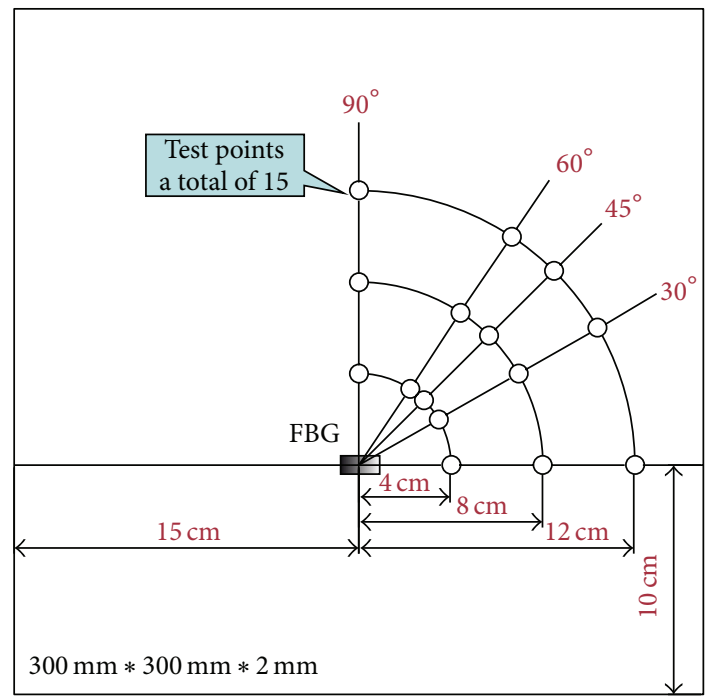

FIGURE 2: Layout of test points and fiber Bragg grating.

In the experiment, longitudinal wave forms lame wave by incident into the plate at a certain angle. This procedure needs the cooperation of the wedge. The mode of lamb wave is different excited by different angles of the wedge. Therefore, the selection of the longitudinal wave incidence angle is critical, due to the limitations of the experimental conditions, just 30 degrees and 45 degrees have been chosen to this incident to identify a more appropriate incident angle on steel plate. It can be seen from Figure 5, when the angle of the incidence is $30^{\circ}$, that the sensitivity of the signal is more strong. Therefore, we chose a wedge of $30^{\circ}$. It can be obviously seen from Figure 6 that the relationship of the ultrasonic intensity on different materials is aluminum $>$ steel $>$ copper. 


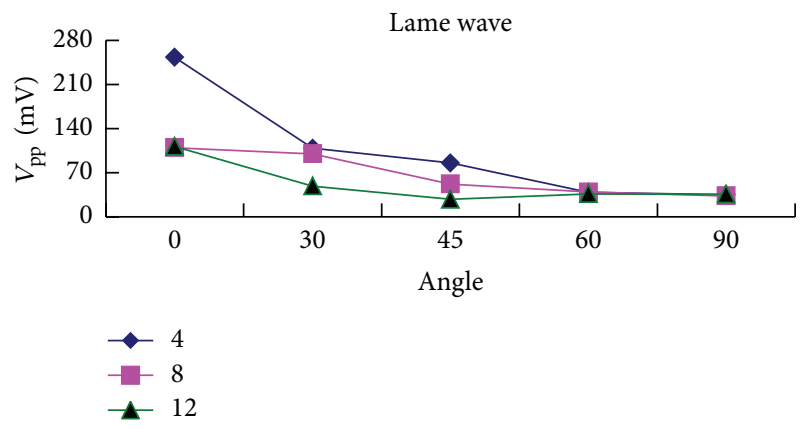

FIGURE 3: Changing curve of ultrasonic intensity on aluminum plate by lame wave.

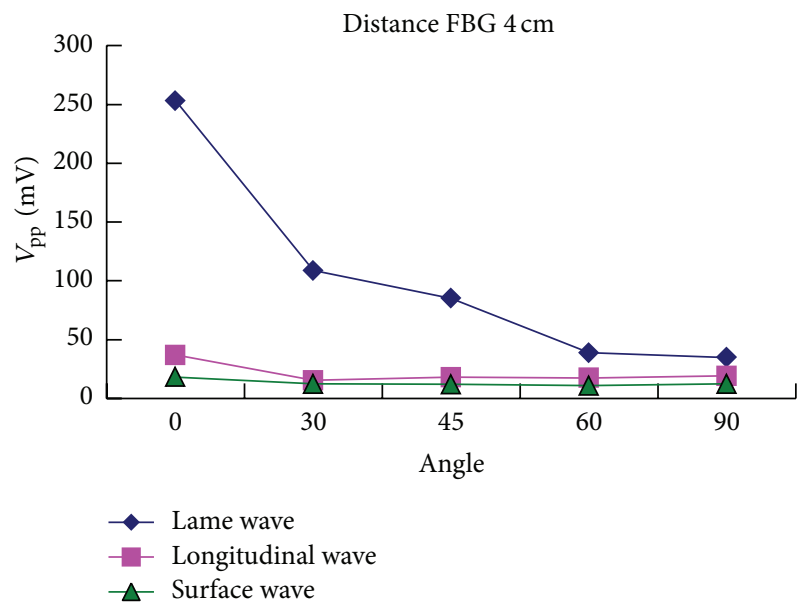

FIGURE 4: Changing curve of ultrasonic intensity on aluminum plate by different waves.

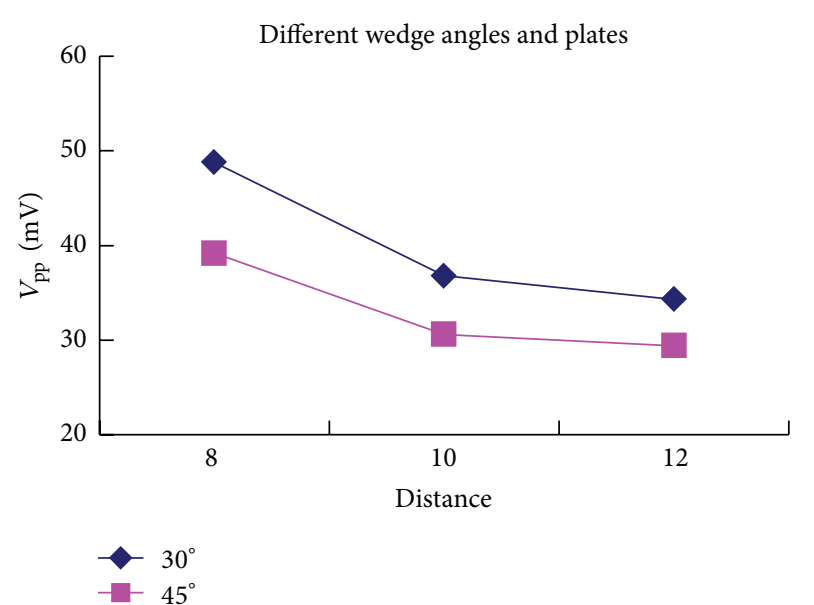

FIGURE 5: Changing curve of ultrasonic intensity on steel plate in different wedge angles.

2.2. The Experimental Analysis on Structure Damage. It can be concluded from the study above that the ultrasonic intensity on aluminum plate is the biggest and lamest wave excitation and that the excitation angle of $30^{\circ}$ is the best. When ultrasonic excitation direction coincides with the axial direction of FBG, the FBG of ultrasonic intensity is the strongest, and the angle is greatly, weakened more obviously. The more

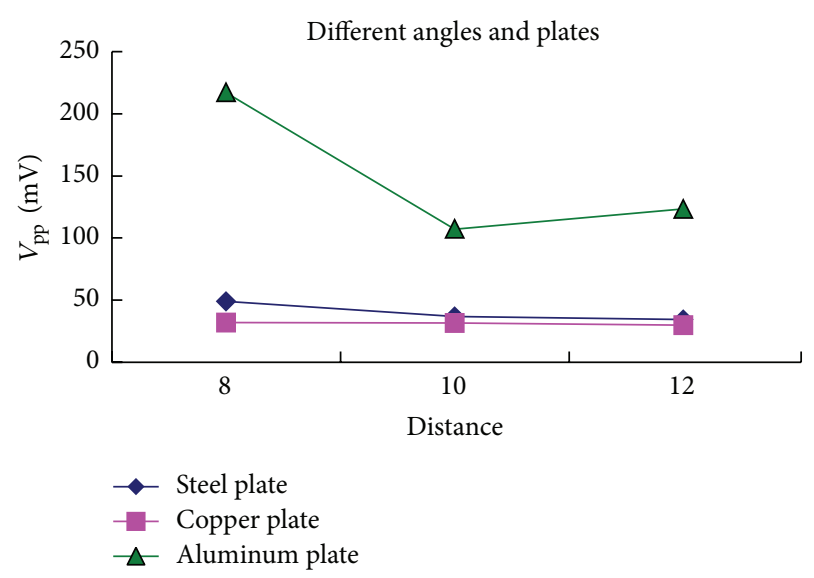

FIGURE 6: Changing curve of ultrasonic intensity on different plates in $30^{\circ}$.

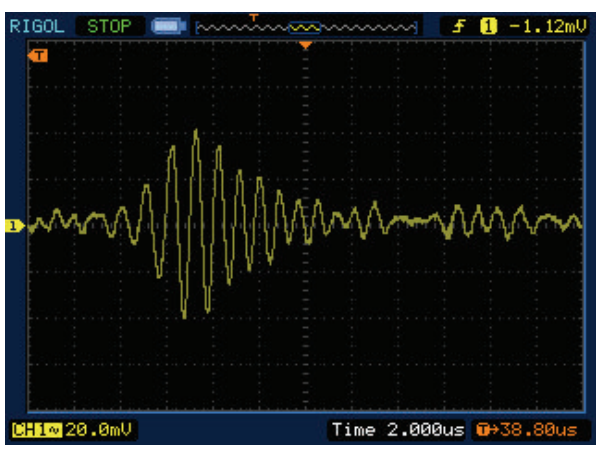

FIgURE 7: The waveform without damage.

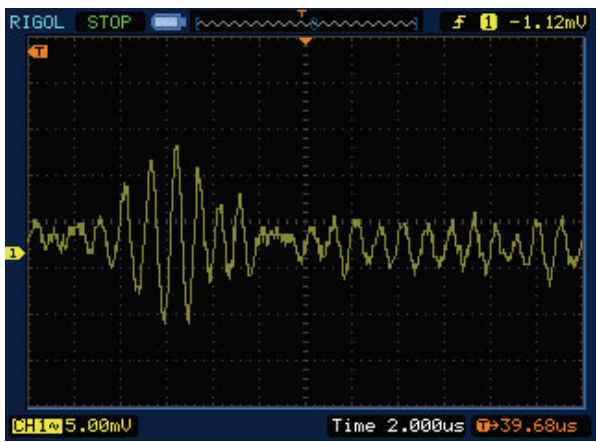

FIgURE 8: The waveform with damage.

the distance between ultrasonic and FBG, the more signal received by FBG is stronger.

Selecting aluminum plate as object, lame wave incidence, the incidence angle of $30^{\circ}$, and designing $6 \mathrm{~mm}$ diameter holes departing $8 \mathrm{~cm}$ from the axis of fiber grating, we can collect the experimental data of the pilot, which is $4 \mathrm{~cm}$ distant from FBG along the axial direction of FBG. It can be found from Figures 7 and 8, in the condition of the defective and nondefective, the waveform of the received signal is substantially different and a wave packet can be seen obviously. Seeking the $V_{\mathrm{pp}}$ average from many experiments, the $V_{\mathrm{pp}}$ of the signal is $133.9 \mathrm{mV}$ in the absence of defects, when defective, 


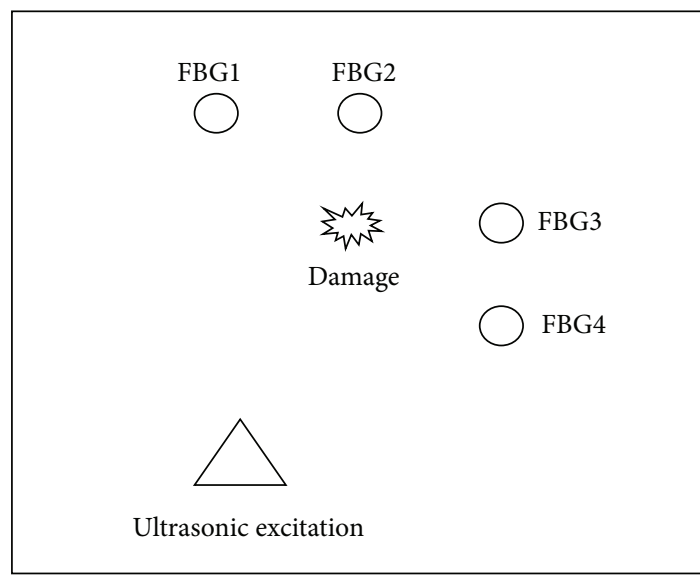

FIGURE 9: Experiment schematic of damage detection.

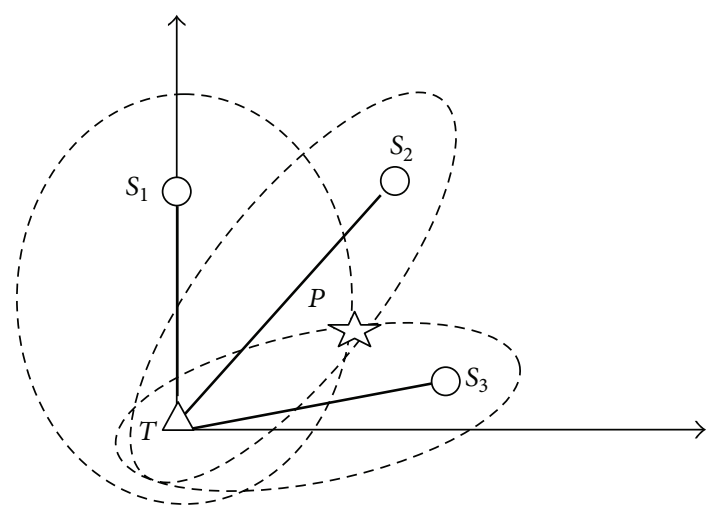

FIGURE 10: Schematic diagram of ellipse detection algorithm.

the FBG of the received ultrasonic intensity is significantly reduced, and the voltage of $V_{\mathrm{pp}}$ is only $44.2 \mathrm{mV}$. It is suggested that the structure damage detection is feasible by FBG.

\section{Damage Location Algorithm}

The experiment schematic of ultrasonic excitation (FBGs) damage detection system is shown in Figure 9, including four distributed optical fiber gratings.

Ultrasonic probe generates ultrasonic signal in plate structure. If damage does not exist in the board structure, sensor only receives health waveform from the ultrasonic probe. When damage exists in the board structure, sensor will receive not only health waveform from the ultrasonic probe, but also emitting wave component produced by the damage, surrounding that which we analyze of the received sensing signal and get information about structural damage.

Figure 10 demonstrates the schematic ellipse algorithm for structural damage detection, in which three small circles represent three fiber Bragg grating sensors, triangle represents an ultrasonic probe, five-pointed star represents damage. Excitation probe is set as the origin of coordinates, and three sensors are located at $S_{1}, S_{2}$, and $S_{3}$. Supposing that distance from excitation probe to one sensor is $d_{i}$, the distance between the spacing of damage and excitation and the spacing of damage and sensor is $l_{i}$.

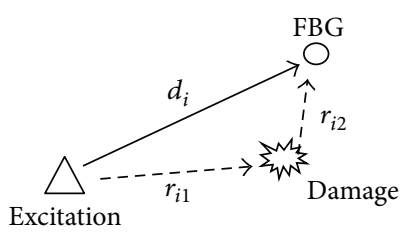

FIGURE 11: Signal propagation path.

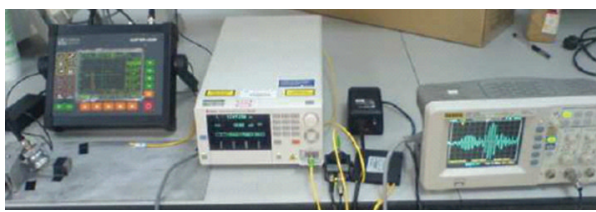

Figure 12: The experimental apparatus.

According to the definition of the ellipse with excitation probe and a fiber Bragg grating sensor as the focus, damage point lies on the elliptical circumference. It is impossible to determine the damage location only by one ellipse. Therefore, we need more fiber Bragg gratings to draw the second or the third ellipse. The intersection of these ellipses is the damaged position.

The basic idea of ellipse algorithm, which has the advantages of simple geometry based on the assumption, is how to determine the delay time of signal transmission from the excitation source to the sensor under the reflection effect of the damage. According to the propagation speed, we can calculate the distance after obtaining the delay time.

Elliptic equation is

$$
\frac{x^{2}}{a_{i}^{2}}+\frac{y^{2}}{b_{i}^{2}}=1, \quad(i=1,2,3) .
$$

The related parameters are defined as

$$
a_{i}=\frac{l_{i}}{2}, \quad b_{i}=\sqrt{\left(\frac{l_{i}}{2}\right)^{2}-\left(\frac{d_{i}}{2}\right)^{2}} .
$$

Therefore, we need three distance parameters to determine the elliptic equation, the first distance $d_{i}$ is from the excitation source to every sensor, the second distance $r_{i 1}$ is from the excitation source to the damaged position, and the third distance $r_{i 2}$ is from the damaged position to the sensor. According to the definition of the ellipse, the distance sum of $r_{i 1}$ plugging $r_{i 2}$ is $l_{i}$.

Figure 11 is signal propagation path. Assumption of $t_{i 1}$ for arrival time of health sensing signals in plate structure and $t_{i 2}$ for arrival time of sensing signals in damaged plate structure, the delay time can be expressed as

$$
\Delta t_{i}=t_{i 2}-t_{i 1}=\frac{r_{i 1}}{v_{0}}+\frac{r_{i 2}}{v_{1}}-\frac{d_{i}}{v_{0}}, \quad(i=1,2,3) .
$$

The related parameter $v_{0}$ is the velocity signal under health mode, while $v_{1}$ is the velocity signal under damaged mode through the damaged plate structure. Because the parameter $v_{1}$ is close to $v_{0}$, we can take the same speed parameters in the calculation process. 


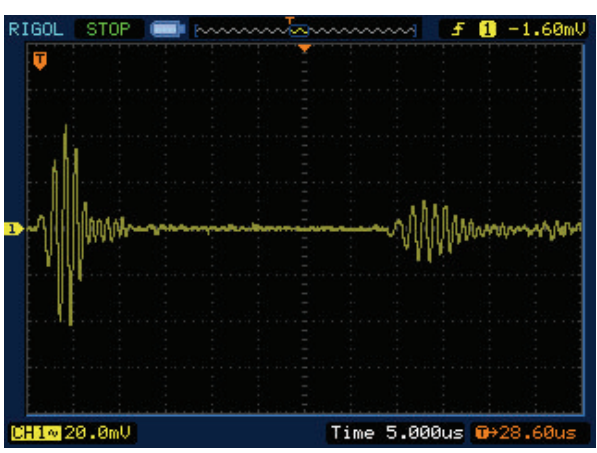

(a) Health waveform

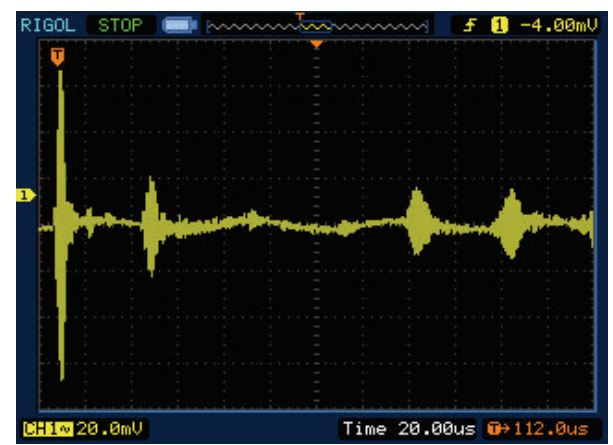

(b) Damage waveform

FIGURE 13: The classical waveform diagram.

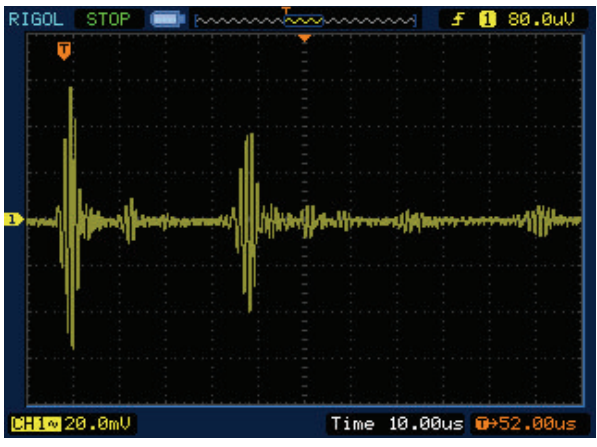

(a) Health waveform

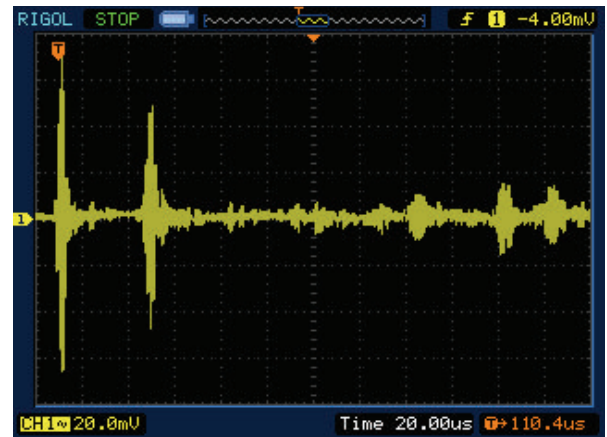

(b) Damage waveform

FIGURE 14: The waveform diagram of the special position.

\section{Analysis of Damage Detection Based on the Test Data}

Based on the above research, the monitoring system of the ultrasonic damage detector was shown in Figure 12, using ultrasonic flaw detector as the signal generator with sensors based on four FBGs, two of which were arranged in a straight line spacing of $50 \mathrm{~mm}$ with the other two on the other line. Ultrasonic signals from ultrasonic probe about a distance of $100 \mathrm{~mm}$ from FBG, were transmitted to the plate structure through the coupling agent, and FBGs pasted on the board structure detected by the ultrasonic strain. The center wavelengths of the FBGs were $1303 \mathrm{~nm}$ and the center frequency of ultrasonic probe was $1 \mathrm{MHZ}$ with the $30^{\circ}$ wedge angle. The demodulation system was to demodulate the FBG reflection wavelength, which would be displayed on a digital oscilloscope display after the photoelectric conversion which was adjusted to $10 \mathrm{~dB}$. Type of tunable laser source was SantecTSL-510, photoelectric detector was Thorlabs-PDA-10CS, and digital oscilloscope was RIGOL-DS1102E.

Taking one sensor, whose sensing signals were collected, we analyzed propagation velocity and delay time of ultrasonic transmission. In order to establish the elliptic equation, we needed to know the distance $d_{i}$ and the distance $l_{i}$, which could be calculated according to the time parameters of the output waveform. Typical health waveform obtained by experiment was as shown in Figure 13(a), and damaged waveform was as shown in Figure 13(b) when the hole diameter of damage was $6 \mathrm{~mm}$. According to the comparison of the waveform parameters shown in Figure 13, the elliptic equation was established. Then, the second elliptic equation was established by another sensor using the same method. Then, we would get the intersection of the ellipses, which was the damage position.

When ultrasonic acoustic axis and optical fiber grating were laid on a line, waveform under damaged mode had no obvious change compared with waveform under health mode, as shown in Figure 14. So, it was very important to select the appropriate incentive points of the ultrasonic excitation source.

The estimation method of delay time for damage analysis had the advantages of simple principle and convenient operation, but estimation accuracy of time delay was limited because the differential signals amplitude between health signal and damage signal obtained were weak.

\section{Conclusion}

This paper had researched the ultrasonic excitation fiber grating sensing system and proposed the location algorithm based on elliptic technology for damage detection, taking the board structure as the object. Firstly, the basic characteristics of fiber Bragg grating sensing signals under ultrasonic excitation were analyzed with different excitation parameters, 
then the damage location algorithm was studied, which had the advantages of simple operation. Finally, the experimental equipments based on ultrasonic excitation-fiber Bragg grating detection were set up. The experimental phenomenon showed that the estimation method of the location algorithm based on elliptic technology was simple, but the accuracy of location detection was limited due to the change of ultrasonic wave mode. In future work, we can try to reduce the frequency of ultrasound excitation in order to improve the positioning accuracy.

\section{Acknowledgment}

This paper is funded and supported by the National Natural Science Foundation of China under Grant no. 51075313, namely, "new principle and method of mechanical damage location detection based on ultrasound excitation and distributed fiber Bragg gratings sensing".

\section{References}

[1] S. Muthumari and A. Singh, "Review of various ultrasonic techniques employed in modern industries," International Journal of Engineering Science and Technology, vol. 3, no. 4, pp. 3078-3085, 2011.

[2] H. Tsuda, "A Bragg wavelength-insensitive fiber Bragg grating ultrasound sensing system that uses a broadband light and no optical filter," Sensors, vol. 11, no. 7, pp. 6954-6966, 2011.

[3] H. Tsuda, N. Toyama, K. Urabe, and J. Takatsubo, "Impact damage detection in CFRP using fiber Bragg gratings," Smart Materials and Structures, vol. 13, no. 4, pp. 719-724, 2004.

[4] B. Culshaw, G. Thursby, D. Betz, and B. Sorazu, "The detection of ultrasound using fiber-optic sensors," IEEE Sensors Journal, vol. 8, no. 7, pp. 1360-1367, 2008.

[5] H. Tsuda, K. Kumakura, and S. Ogihara, "Ultrasonic sensitivity of strain-insensitive fiber Bragg grating sensors and evaluation of ultrasound-induced strain," Sensors, vol. 10, no. 12, pp. 1124811258,2010

[6] B.-W. Jang, S.-O. Park, Y.-G. Lee, C.-G. Kim, and C.-Y. Park, "Detection of impact damage in composite structures using high speed FBG interrogator," Advanced Composite Materials, vol. 21, no. 1, pp. 29-44, 2012.

[7] B.-W. Jang, Y.-G. Lee, J.-H. Kim, Y.-Y. Kim, and C.-G. Kim, "Real-time impact identification algorithm for composite structures using fiber Bragg grating sensors," Structural Control and Health Monitoring, vol. 19, no. 7, pp. 580-591, 2012. 

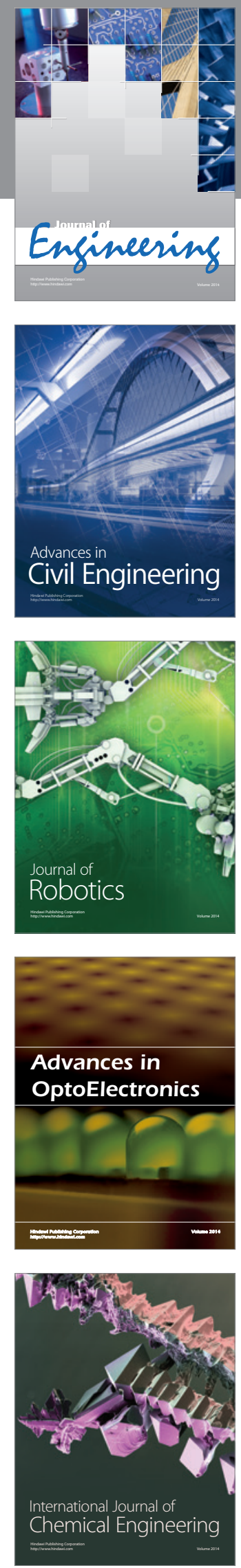

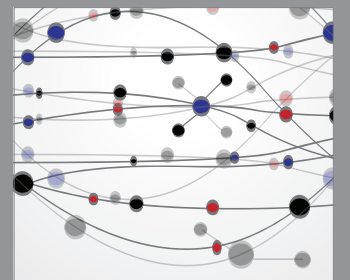

The Scientific World Journal
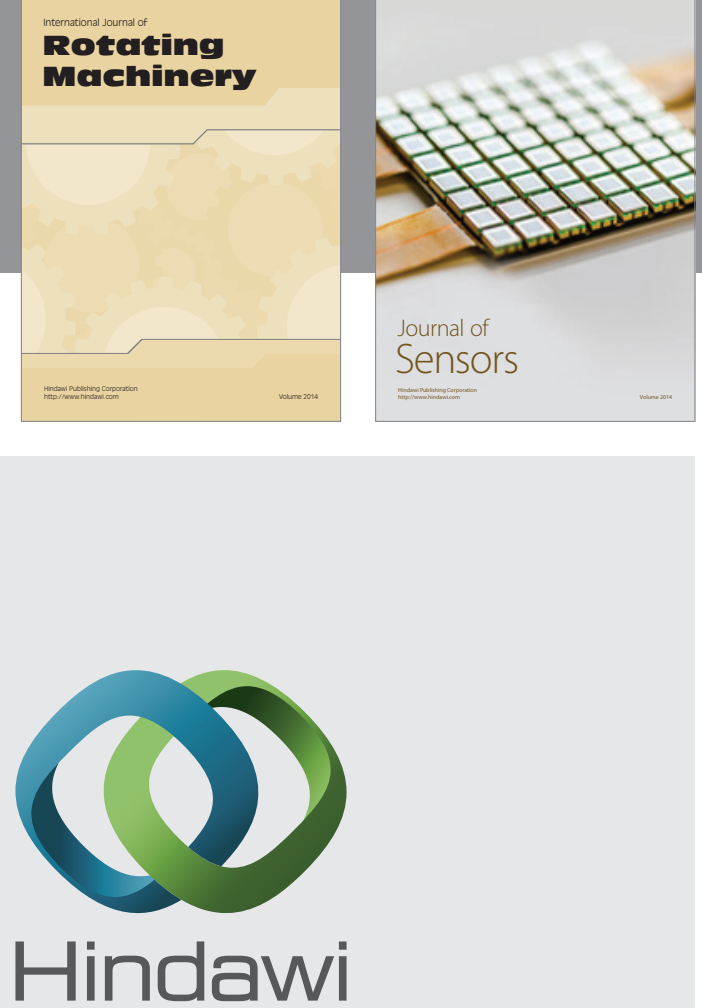

Submit your manuscripts at http://www.hindawi.com
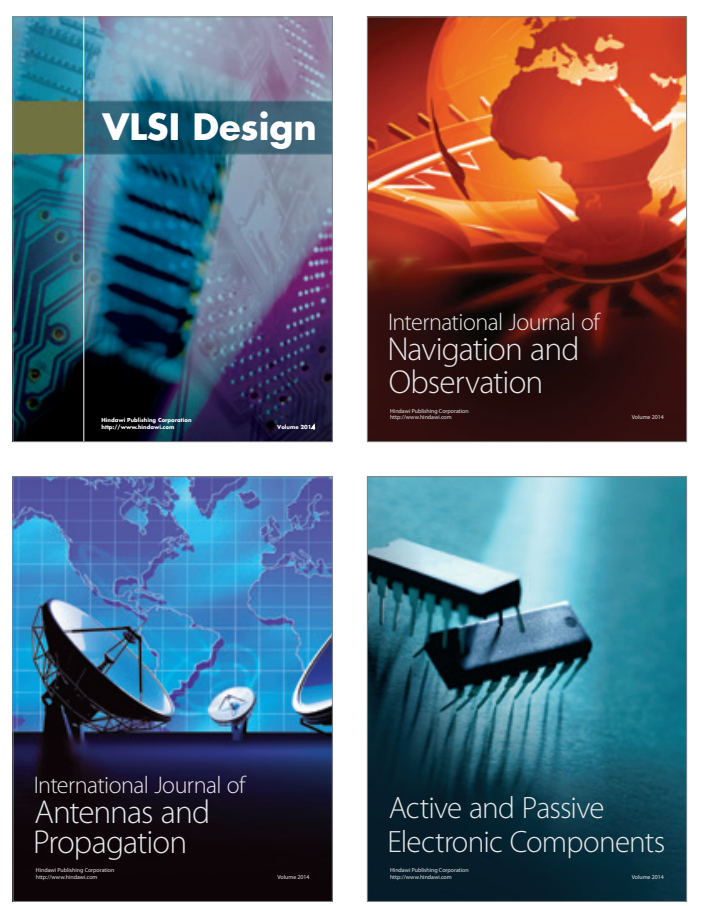
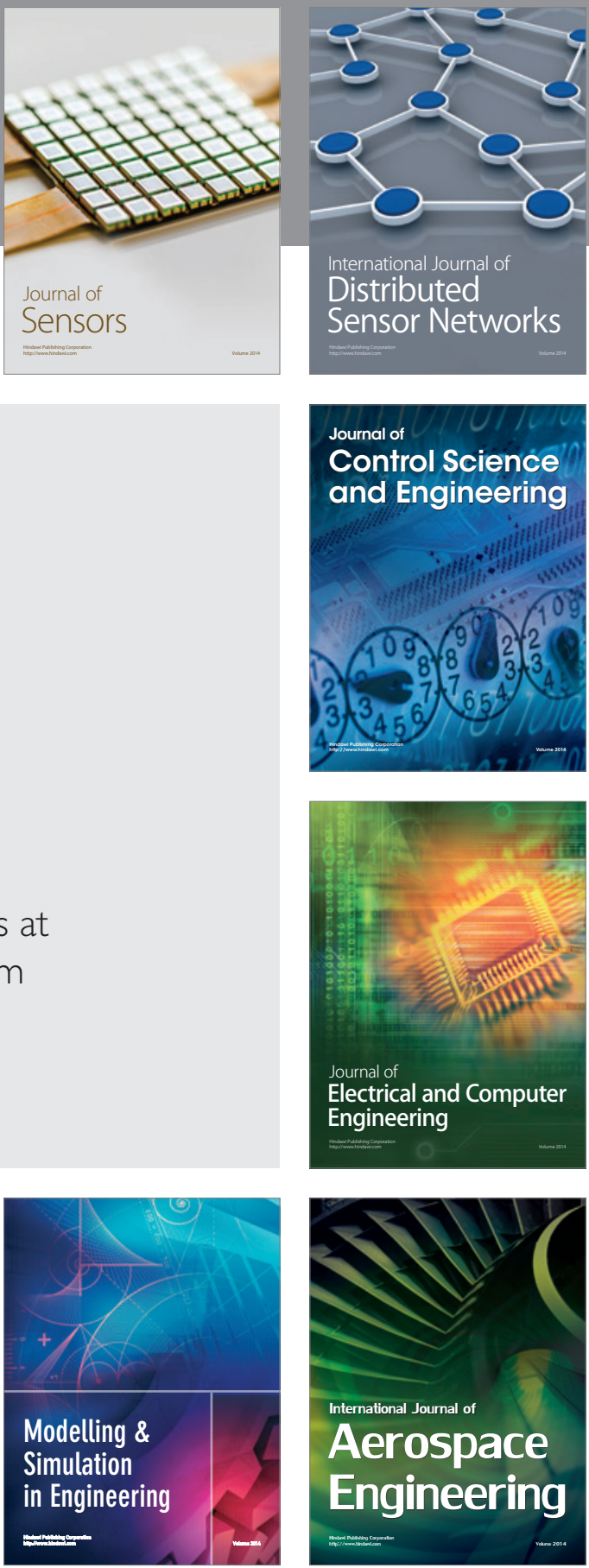

Journal of

Control Science

and Engineering
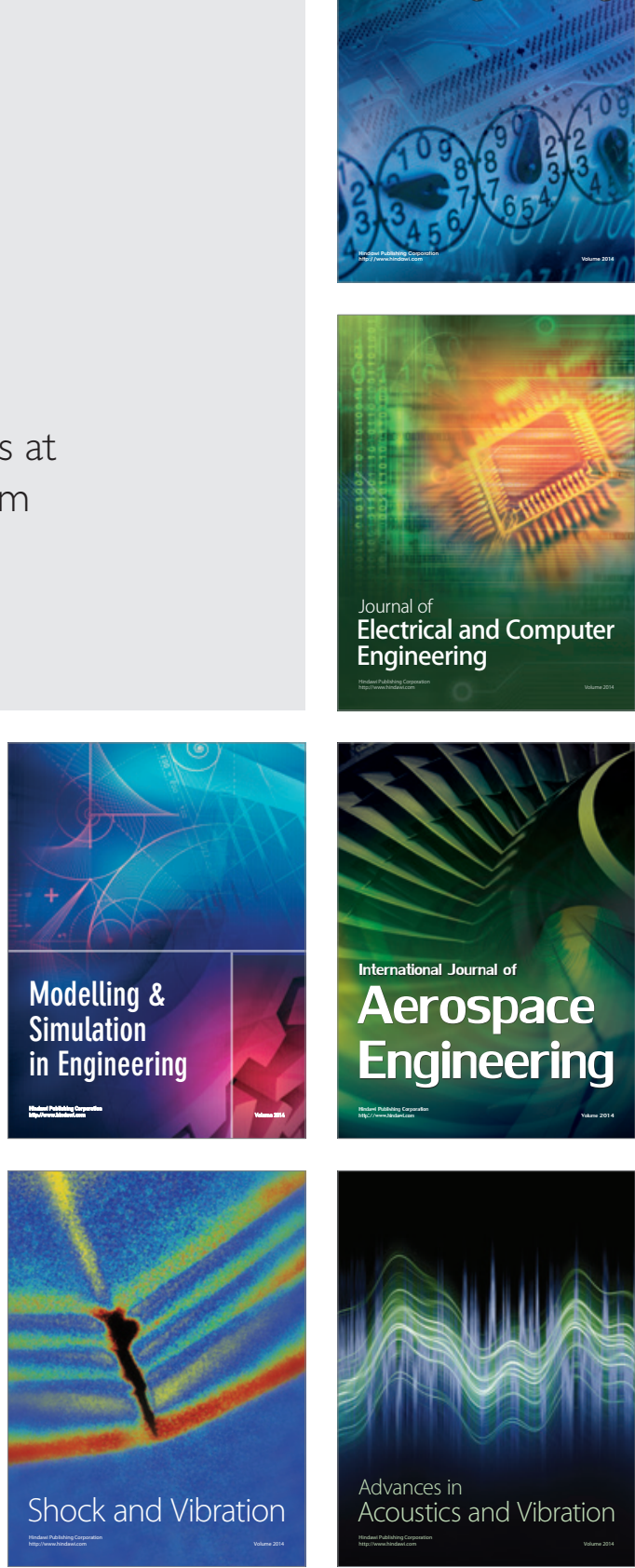